\title{
Dim Baryons in the Cosmic Web
}

\author{
Chris D. Impey ${ }^{1}$ \\ ${ }^{1}$ Department of Astronomy, University of Arizona, Tucson, AZ 85721, USA \\ email: cimpey@as.arizona.edu
}

\begin{abstract}
The distribution of baryons beyond galaxies is descibed. The majority of the baryons, which represent $4 \%$ of the cosmic mass and energy budget, lie far from individual galaxies in the diffuse intergalactic medium (IGM). Many of these baryons are in a warm phase that can be probed by quasar absorption in the Lyman- $\alpha$ line of hydrogen. The mature field of quasar spectroscopy can diagnose the location, physical state, metallicity, and general geometry of this gas, which is called the "cosmic web." The remainder of the gas is kept very hot by infall and shocks and is mostly in higher density regions such as filaments, groups and clusters. The hot gas is only detectable via X-rays and the absorption of highly ionized species of heavy elements. The baryons in low density regions of space are excellent tracers of underlying dark matter. The evolution of the cosmic web indicates where to look for the baryons in collapsed objects but the overall inefficiency of galaxy formation has conspired to keep most baryons dark.
\end{abstract}

Keywords. intergalactic medium, quasars: absorption lines, dark matter, large-scale structure of universe, cosmology: observations, X-rays: diffuse background, atomic processes

\section{Introduction}

In the cosmic census of matter and energy, baryons are a small component, almost an afterthought left over from symmetry-breaking processes at the era of unification in the tiny fractions of a second after the big bang. Baryons are outnumbered six to one by the weakly interacting massive particles that form dark matter, and by a factor of a hundred million by the sea of low energy photons leftover from the early hot phase of the universe. We are naturally fond of them, since they make up our bodies, and the Earth, and the $10^{22}$ stars with their attendant planets and (presumed) biological organisms that form the baryonic universe.

Despite recent progress in cosmology, we remain largely ignorant of the location of baryons in the local universe. In a formal sense, the baryons are not "lost" or "missing" they have merely been mislaid. We knew where they were after the first few minutes, when big bang nucleosynthesis models give good agreement with the abundance of several species of light elements (Coc 2006). We also knew where they were after 300,000 years, when they imprinted on the microwave background radiation (Kamionkowski et al. 1995). We still could locate them 2-3 billion years after the big bang, when few of them had yet migrated from intergalactic space to the deep dark matter potentials that would undergo non-linear collapse and galaxy formation (Rauch et al. 1997). But over the last $75 \%$ of the age of the universe, the baryons have faded (or, in many cases, brightened) from view, leaving us to parse and study for our amusement the mere 10-15\% that reside in the galaxies of our surveys and catalogs.

Most baryons are hot and diffuse. This review will summarize observational approaches to detect baryons in the intergalactic medium (IGM). The primary tool is quasar spectroscopy using absorption by hydrogen in Lyman- $\alpha$ lines and by metals in the form of highly ionized species that are visible at ultraviolet or X-ray wavelengths. Our expectations are shaped by recent cosmological hydrodynamic simulations. 

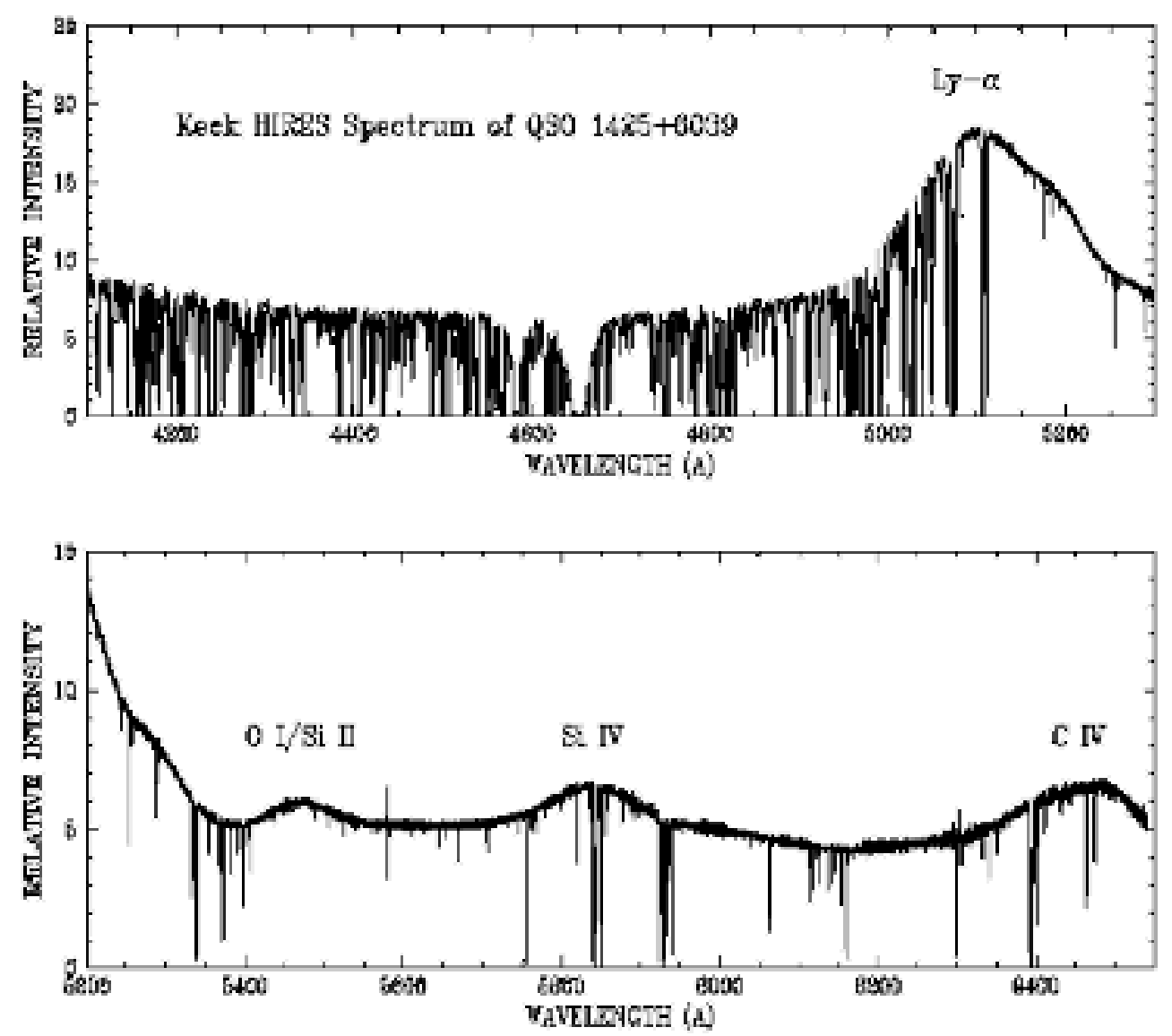

Figure 1. Example of the high quality and high SNR quasar spectra that can be made using large telescopes, in this case the Keck 10-m on Mauna Kea. Blueward of Lyman- $\alpha$ emission is the thicket of narrow absorption features from the Lyman- $\alpha$ "forest." Redward are metal lines associated with this generally metal-poor intergalactic gas. Spectrum courtesy of Wallace Sargent, California Institute of Technology.

\section{Probing the Evolving IGM}

Living as we do in a local overdensity of the universe it is worth recalling how amazingly empty the space between galaxies is. To tether this with an everyday analogy imagine taking a cubic centimeter of the air you are breathing and stretching one dimension of it by a megaparsec, to beyond Andromeda: that is the intergalactic medium. The extremely low density is one reason baryons in the IGM are hard to detect. Quasars act as distant flashlights to give 1D maps of velocity versus transmission or opacity, where the optical depth derives from the very small mass fraction of the gas that is neutral. The best quality data have extraordinary sensitivity; Figure 1 shows a case where the weakest absorbers have a neutral hydrogen column of only $10^{11.5}$ atoms $\mathrm{cm}^{-2}$.

The universe has expanded by an order of magnitude since it was a simple mixture of primordial hydrogen and helium. Gas has been cycled through galaxies for 12 Gyr and the IGM has been enriched inhomogeneously at all redshifts. This can be seen, for 


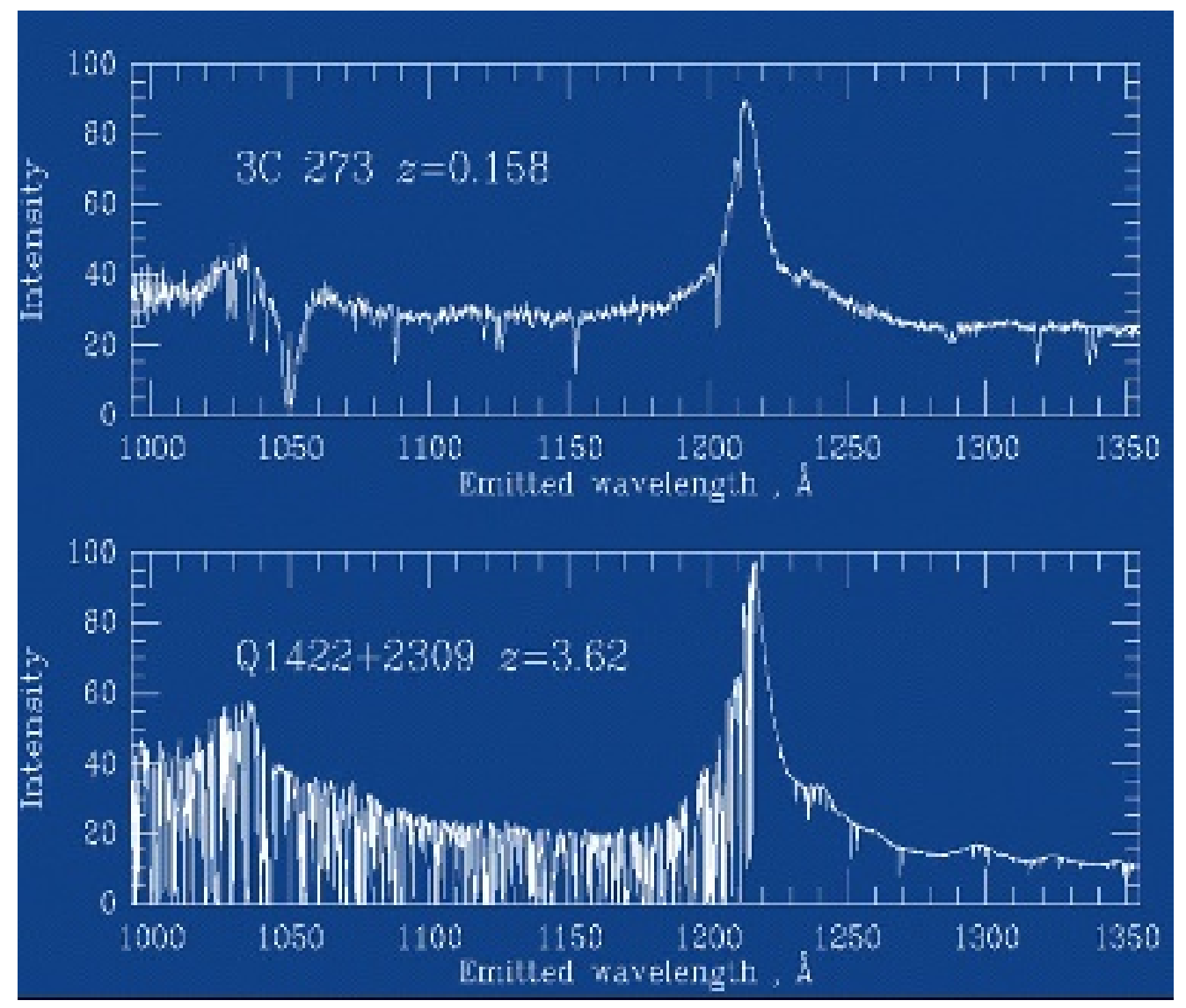

Figure 2. Two spectra that dramatically show the evolution of the IGM. At the top is the bright low redshift quasar $3 \mathrm{C} 273$, showing modest levels of absorption. The bottom spectrum traces a path through the universe when it was 5 times hotter and 100 times denser, and there is Lyman- $\alpha$ opacity at almost every pixel. Spectra courtesy of William Keel, University of Alabama.

example, in the large range of $-3<\log [\mathrm{Fe} / \mathrm{H}]<-0.5$ for Lyman limit and damped Lyman- $\alpha$ quasar absorbers, where the envelope of enrichment changes very slightly with redshift (Prochaska et al. 2007). At low redshift, $\log [\mathrm{C} / \mathrm{H}]$ is observed to vary from -1.2 in a cluster environment to $<-2.3$ in a lower density region (Shull et al. 1998; Tripp et al. 2002). In the future, O VI in the warm-hot IGM will also be useful as a metallicity tracer, although the modelling is complex for a species that can be both photoionized and collisionally ionized. The evolution of the IGM is most clearly seen in the dramatic change in opacity and absorber density going from high to low redshift (Figure 2).

Other issues relate to the way in which metals are recycled into the IGM. This complex process is driven by low mass galaxies, which is still a very poorly understood population since there are very few cataloged field galaxies with either $M_{\mathrm{B}}<-16$ or $M_{\mathrm{HI}}<10^{8} \mathrm{M}_{\odot}$ (Zwaan et al. 2005). Galactic winds carry metals out into the IGM, and in extreme cases the mass outflow rate can equal the star formation rate. Detailed studies show that about half the supernova kinetic energy escapes to heat the IGM, and that the gas is mostly bound for $v_{\text {circ }}<80 \mathrm{kms}^{-1}$ (Martin 2006). Thus, it is likely that baryonic blowout can explain the deficit of visible counterparts to the abundant low mass dark matter halos that are predicted by heirachical clustering (Moore et al. 1999). 


\section{Learning from Hydrodynamic Simulations}

Simulations that add gas dynamics to gravity have achieved great success in showing the complexity of large scale structure and in framing issues that might be amenable to observation. The best simulations have cooling by metals and feedback from AGN incorporated, and they may include up to a billion particles. There is something seductive about unleashing the expanding universe in a "box" and sitting back while it evolves, then using simple tools to extract an almost infinite number of spectra from which it's easy to measure gas temperatures, overdensities, line profiles, column densities, and metallicities. Before we get too giddy, however, we recall that the simulations are only as good as their input physics, and the large but finite number of particles still leads to tradeoffs between fidelity for large scale structure and the inner regions of galaxy-scale halos. The general sense of the evolution is that gas in the cosmic web departs from Hubble flow to fall onto sheets and filaments, and then into galaxies and clusters (Figure 3).

Baryons range over seven orders of magnitude in temperature and density, from galaxy disks to the hot intra-cluster medium. Hydrodynamic simulations have illuminated the likely location and physical state of baryons that are too diffuse or hot to be detected using optical techniques. At high redshift ( $z \sim 2$ or above), when structure formation has not proceeded very far and almost all baryons are in a diffuse, filamentary IGM, the fluctuating Gunn-Peterson approximation provides a simple prescription for the physical state. The scaling of HI opacity goes as $\tau_{\mathrm{HI}} \propto \rho_{\mathrm{DM}}^{1.6-1.8}$ (Davé et al. 2001), and the amount of photoionization of the highly ionized medium, which is a free parameter in the simulations, is set by measurements of the proximity effect (Scott et al. 2002). As the universe expands, gravity and shocks create a caustic network of warm/hot gas (WHIM) with groups and clusters of galaxies at the nodes. Meanwhile, the expansion creates a cooler medium that is in photoionized equilibrium at $\sim 10^{4} \mathrm{~K}$.

By low redshift, this simplicity has dissipated. Local baryons are found in a wide range of environments and they are subject to the non-linear physics of shocks and gravitational collapse (Figure 4). Spectroscopy of the Lyman- $\alpha$ forest is a mature technique, but the shocks are not resolved in most spectra and the $1 \mathrm{D}$ velocity information non-uniquely codes thermal motion, inflow, outflow, and winds. The 1D structure is often a poor quide to the 3D geometry. A good example of the challenges of the field is the difficult of measuring metallicity with a few tracers. The IGM is unlikely to be any less complex than the local ISM. Hydrodynamic simulations "predict" that local baryons are divided roughly equally between those that condensed into galaxies, those that form the cosmic web of structure delineated by the Lyman- $\alpha$ absorbers, and those that form the caustic network traced by the warm-hot medium (Davé et al. 2001).

\section{Detecting the Warm-Hot IGM}

Observational handles on the complex, multi-phase IGM are still fairly primitive. The warm/hot medium is most readily seen in X-ray emission and O VI absorption, but this data is still sparse and difficult to interpret. The Local Group may provide the best local analog of the temperature, density and filling factor of the hot gas (Sembach et al. 2003). The relatively recent detection of the warm-hot phase through the O VI (Tripp et al. 2007) and O VIII tracers (Wang et al. 2005) is very exciting. Early evidence indicates that the O VI absorbers have $d N / d z \sim 50(\mathrm{REW}>30 \mathrm{~mA}$ ), with one for every ten Lyman- $\alpha$ absorbers; the O VI absorbers are clustered and closely follow the galaxy distribution. Much closer to home, the covering factor of O VI in the extended Galactic 
a)

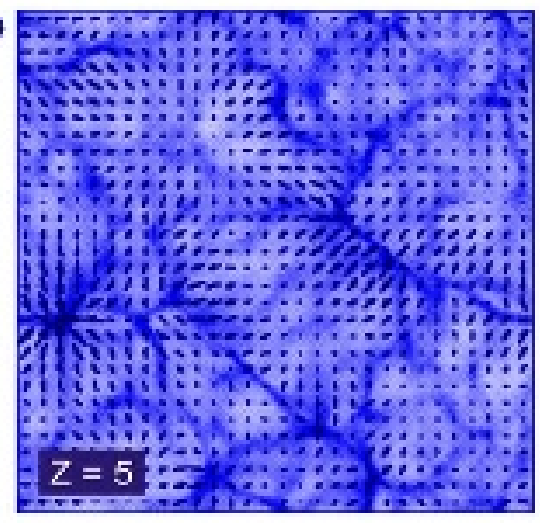

c)

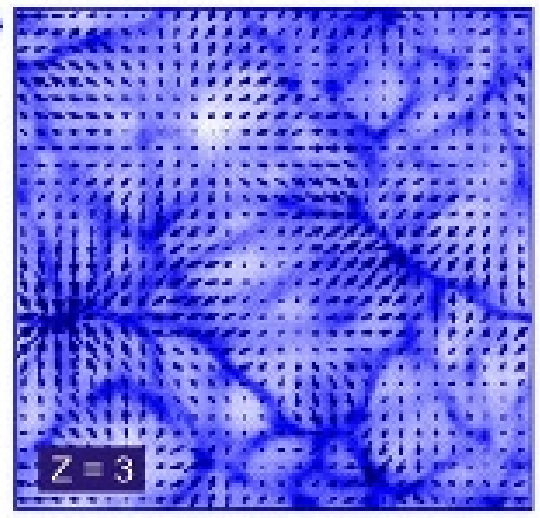

b)

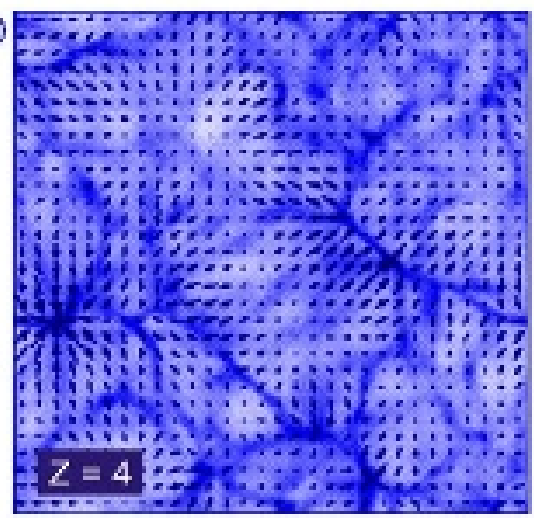

$\log \left(\delta_{\mathbf{b}}\right)$

\section{d)}

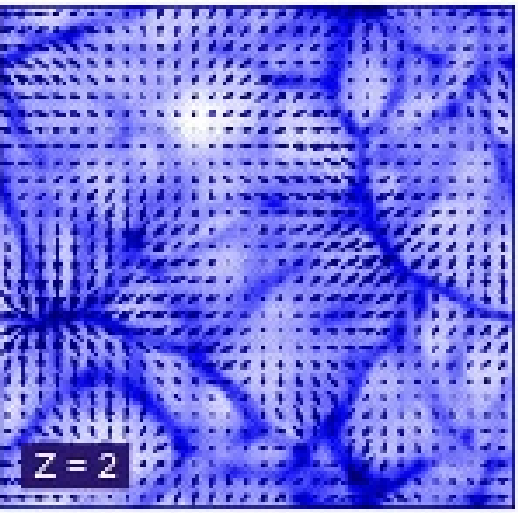

2.5

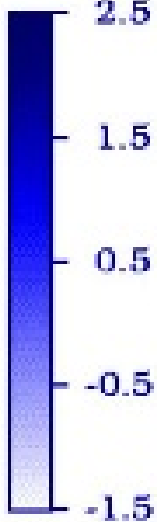

Figure 3. Hydrodynamic simulations of cosmological "fair samples" of the universe show that baryons follow gravity defined by dominant dark matter and so flow away from voids and onto filaments, sheets and putative clusters. The gray scale is baryon overdensity and the arrows show peculiar motions, which imprint on observed spectra as they pass through this "cosmic web" of large scale structure. From Zhang et al. (1997).

halo is observed to be $\sim 80 \%$ (Wakker et al. 2005), contained either in a hot Galactic corona or an extended Local Group medium.

The observational attack on the warm-hot IGM is in its early stages. Data quality is governed by the limited sensitivity of UV and X-ray spectroscopy from space. For example, the BL Lac 2155-304 is so bright in X-rays that it is essentially unique within the volume of its redshift distance, about $10 \mathrm{Gpc}^{3}$. Different tracers reveal different aspects of the highly inhomogeneous IGM. Quasars can probe redshift path but give no measure of absorber size and extent, while deep X-ray imaging will offer maps confused by projection across redshift space. It will be some time before we approach the level of $3 \mathrm{D}$ "tomography" that is now becoming possible with the Lyman- $\alpha$ forest for the cooler IGM gas. Another way to separate the warm-hot and cooler phases uses high resolution spectra to diagnose the temperature via the Doppler parameter distribution of Lyman- $\alpha$ absorbers (Lehner et al. 2007). Simulations indicate that the hottest absorbers sometimes show up in HI, so separating the WHIM from the Lyman- $\alpha$ forest can be tricky.

The first attempts to detect WHIM gas by X-ray absorption have produced a handful of weak detections using very bright AGN as probes (Figure 5). The situation will not improve until a future generation of X-ray telescopes in space is launched. NASA's 


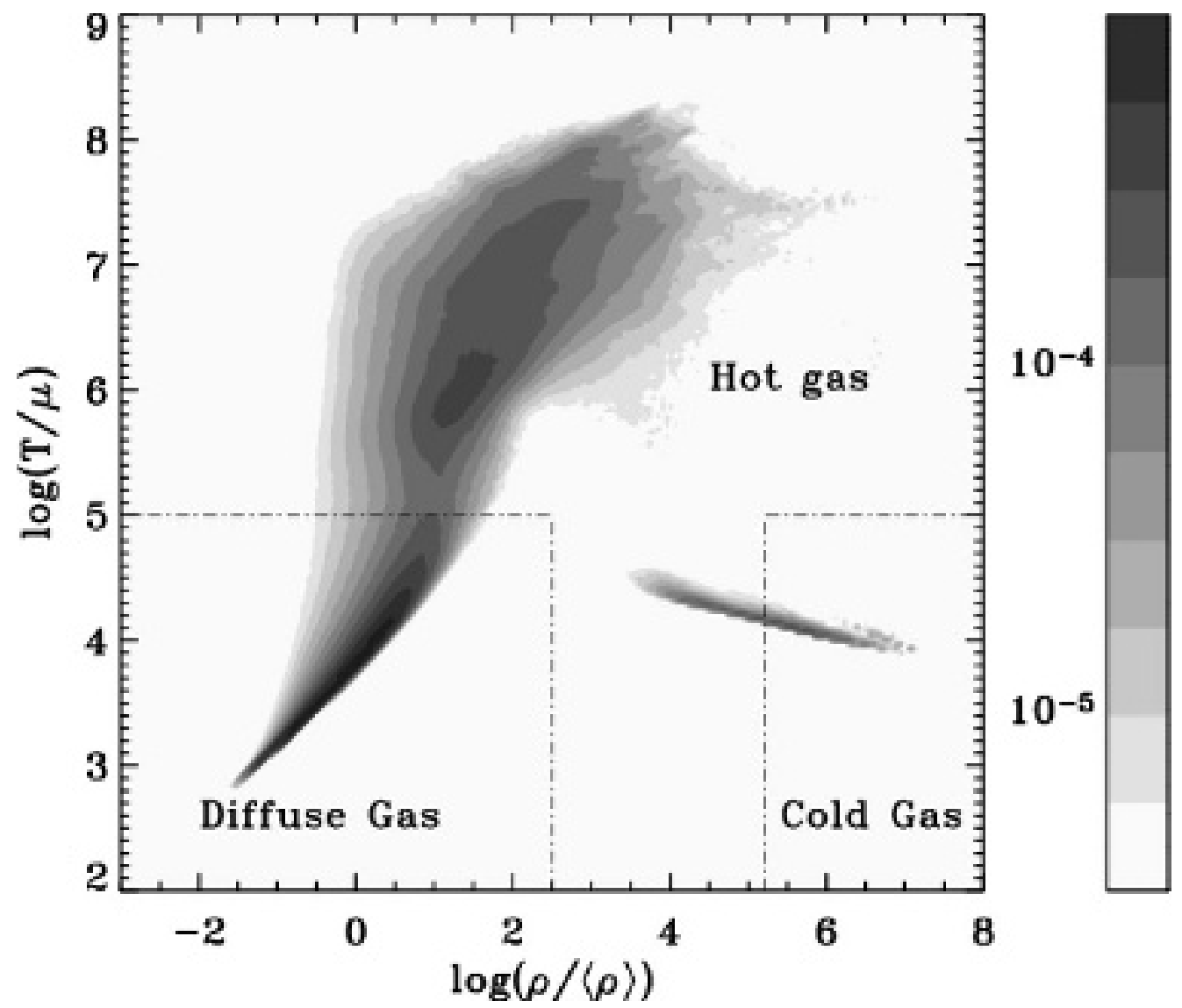

Figure 4. At low redshift, a small fraction of the gas in the IGM has fallen into deep gravitational potential wells and cooled to form galaxies. But most of the gas has been heated as it forms a web of shocked filaments and caustics; Lyman- $\alpha$ absorption can only measure the $\sim 0.01 \%$ of the gas that is in the neutral phase. From Rasera \& Teyssier (2006).

Constellation X will improve on XMM-Newton spectral sensitivity by a factor of ten. The XEUS mission is ESA's successor to XMM-Newton, 100 times more sensitive and due to launch in 2015. Simulations show that XEUS will be able to detect $20 \%$ of all baryons, with unambiguous detections of WHIM filaments in only $100 \mathrm{ksec}$ (Kawahara et al. 2006). A third of its random beams will see both O VII and O VIII, allowed temperature to be measured. Meanwhile, other methods will be use to pursue dim baryons. The final results of WMAP will achieve a solid measurement of the second Doppler peak, and future microwave experiments may be able to trace the bulk of the WHIM through its thermal Sunyaev-Zeldovich effect (Hernandez-Monteagudo et al. 2006). Ingenuity will be needed to snare such an elusive adversary.

\section{Relating Dim Baryons to Large Scale Structure}

Galaxies in the local universe trace out the skeleton of large scale structure, but the remaining baryons have a very complex relationship to the underlying dark matter distribution. Quasar absorption is the most effective way of tracing the baryons in galaxy disks (via damped Lyman- $\alpha$ ), galaxy halos (using highly ionized species like C IV and Mg II), and the the diffuse IGM (using Lyman- $\alpha$ absorbers) using long 1D sightlines (Petitjean 


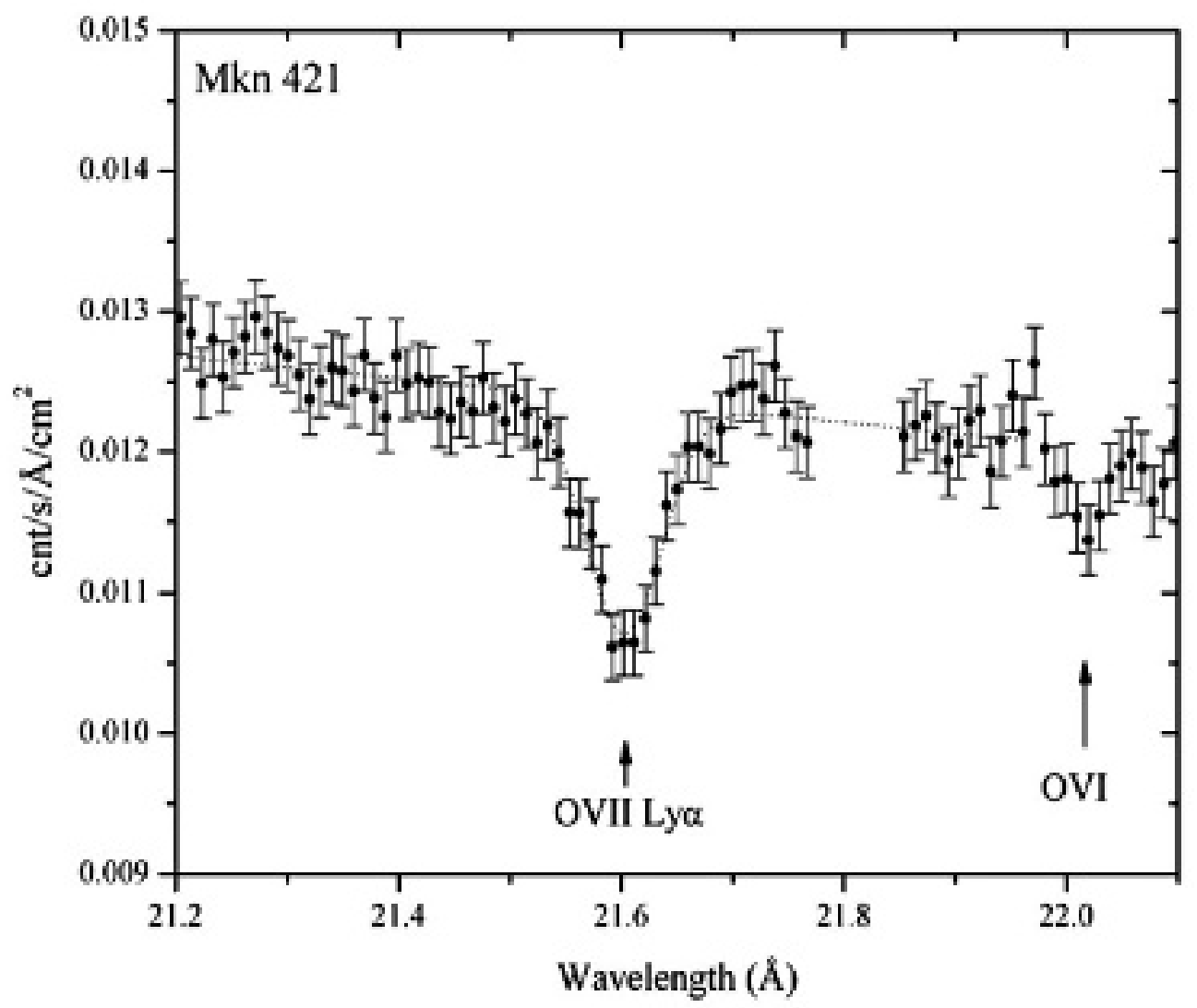

Figure 5. This X-ray spectrum of the bright BL Lac object Mark 421 was obtained with the RGS on XMM-Newton, showing a clear detection of the warm-hot IGM phase in this highly ionized oxygen line. Similar detections will be ten times faster with the upcoming Constellation X mission. From Bregman \& Lloyd-Davis (2007).

\& Charlot 1997). In many cases it is probably not physically meaningful to try and relate individual absorbers to individual galaxies. There is often a Lyman- $\alpha$ absorber a small projected separation from a bright galaxy, but not every absorber has a bright galaxy nearby (Bowen, Pettini, \& Blades 2002). The environment of the high ionization WHIM absorbers is beginning to be explored (Stocke et al. 2006).

Future progress will depend on large statistical studies that allow large samples of absorbers to be related to local galaxy density. Already, there is evidence for Lyman- $\alpha$ clouds in voids, where galaxy formation is extremely inefficient (McLin et al. 2002). It is also possible that absorbers below a column density of $10^{12}$ atoms $\mathrm{cm}^{-2}$ form a kind of intergalactic "froth." At higher columns absorber-absorber correlations are much weaker than galaxy-galaxy correlations (Impey, Petry, \& Flint 1999; Penton et al. 2002). The Colorado group find that a third of all baryons are contained in Lyman- $\alpha$ absorbers down to $10^{13}$ atoms $\mathrm{cm}^{-2}, 5 \%$ of the baryons in absorbers are in voids, in agreement with simulations, and half of the absorbers contain metals with $10 \%$ solar abundance (Figure 6). The typical distance from an absorber to the nearest $L_{*}$ galaxies is $350 \mathrm{kpc}$. It is not possible to get a dense enough packing of sightlines for $3 \mathrm{D}$ "tomography" of the forest but the prospects will improve dramatically with the much-anticipated launch of 


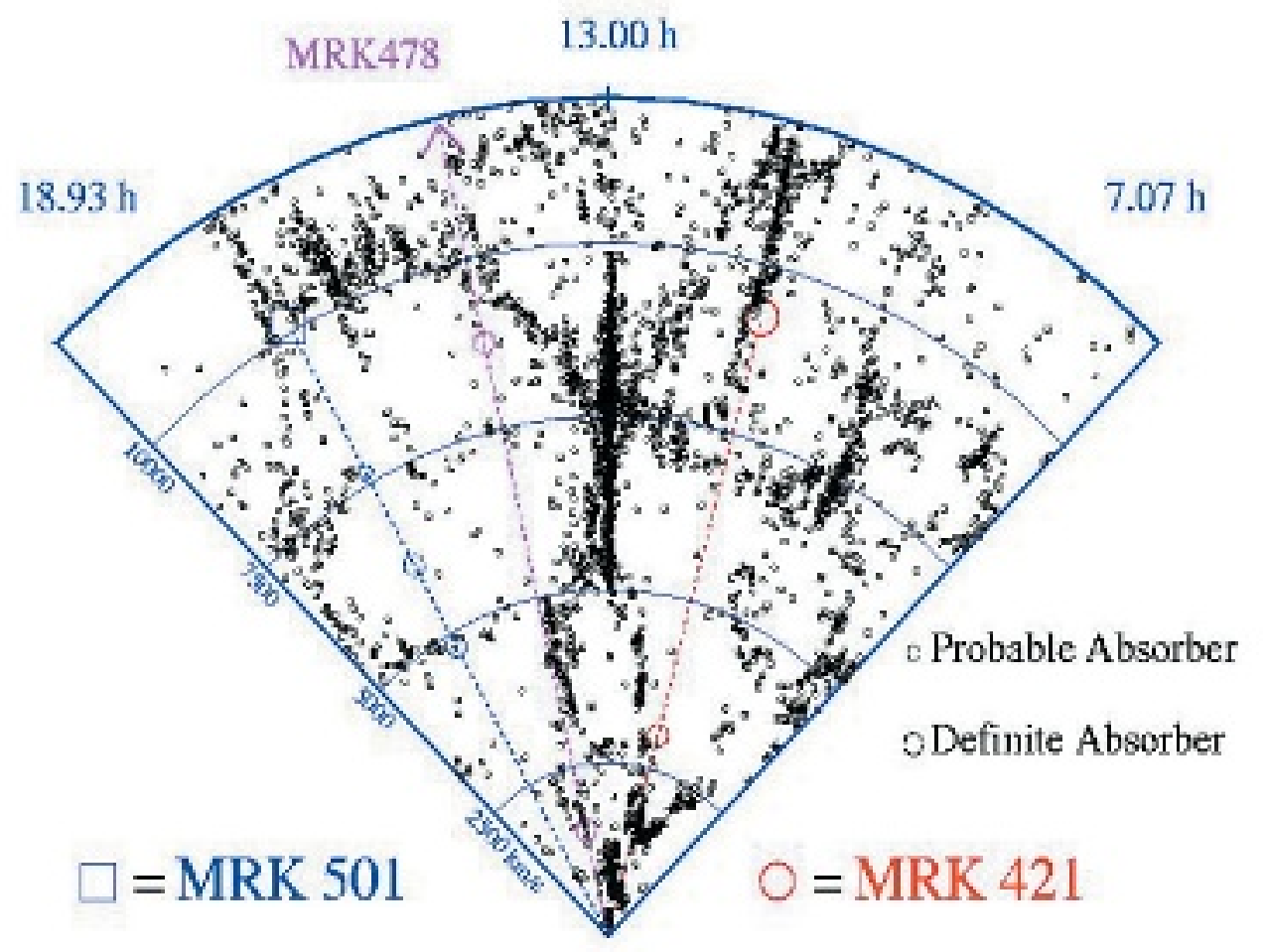

$\alpha$ wedge for $\delta=32.50 \pm 5.0^{\circ}$

Figure 6. The distribution of very low redshift Lyman- $\alpha$ absorbers along three sightlines studied with HST data, compared to a pie chart of galaxy redshifts from the CfA redshift survey. Absorbers tend to avoid clusters and high density regions, and a significant fraction are found in voids. Two of the AGN probes are bright BL Lac Objects. From Penton, Shull \& Stocke (2000).

the Cosmic Origins Spectrograph (COS) in 2008 (Green 2001). High sensitivity data on the low redshift Lyman- $\alpha$ forest can then be combined with the very dense sampling of the $2 \mathrm{dF}$ and SDSS galaxy redshift surveys.

Baryons are the interactive and entertaining side show of the universe; numerical simulations have revealed the way they interact and redistribute themselves within dark matter halos. Cuspy dark matter cores are a generic prediction of CDM, and the jury is still on whether or not the flat rotation curves seen in some dwarf and LSB galaxies are signs that the mass distribution is too flat (McGaugh, Rubin, \& de Blok 2002); triaxial dark matter haloes may yet explain the observations (Hiyashi \& Navarro 2007). The search continues for nearby examples of galaxies that will make the best laboratories for dark matter studies. On larger scales of 1-10 Mpc, the low redshift Lyman- $\alpha$ forest provides the prospect of a direct inversion from opacity to the mass power spectrum, assuming COS observations will give a substantial number of spectra down to a column density that corresponds to a low gas overdensity, where shock heating is not as pervasive and the bias can be understood. 


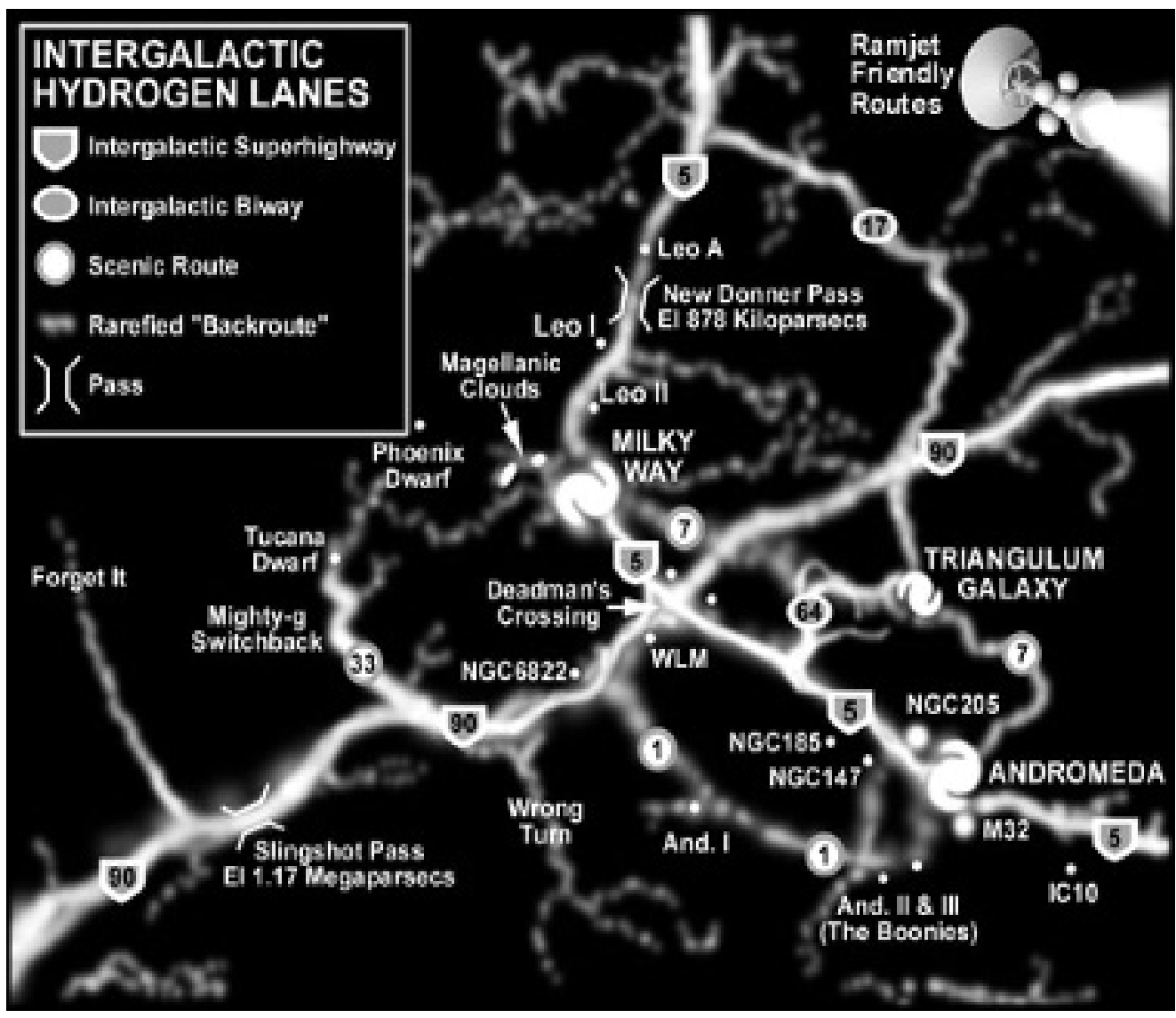

Figure 7. A guide to navigating the complex topology of the IGM. Landmarks and helpful roadsigns are unavailable for most baryons. Courtesy of John Cramer and Wolf Read.

\section{Conclusions}

Baryons continue to be elusive. There is broad concordance around a value of $\Omega_{b}=0.04$ 0.05 from WMAP measurements of the microwave background, light element abundance from comsic nucleosynthesis, and the flux and opacity distribution in the Lyman- $\alpha$ forest at $z=2-4$. Galaxies contain only $10-15 \%$ of this census. The remainder of the baryons are diffusely distributed and dim. About $20 \%$ are in the relatively cool IGM, about $30 \%$ are in gas near galaxies, primarily groups and clusters. The largest fraction, about $40 \%$ are in highly ionized intergalactic gas at temperatures of $10^{5}$ to $10^{6} \mathrm{~K}$. All of these phases have been detected, and absorption using AGN as flashlights has proven to be a powerful tool for characterizing the spatial distribution, metal abundance and temperature of the gas. The Cosmic Origins spectrograph on HST and several planned X-ray missions will increase the observational capability for this kind of work by an order of magnitude.

\section{Acknowledgements}

I acknowledge Craig Foltz, Andy Marble and Cathy Petry, my collaborators and coconspirators in work on the Lyman- $\alpha$ forest. My attendance at this conference was made possible by the UA's Office for International Programs and the IAU. Thanks to Jon Davies and the local organizers for running a tight ship that left little for the more far-flung organizers to do; the tight ship came in handy during a rainy week in Cardiff. 


\section{References}

Bowen, D. B., Pettini, M., \& Blades, C. J. 2002, ApJ, 580, 169

Bregman, J. N., \& Lloyd-Davis, E. J. 2007, ApJ, in press, astro-ph07071699

Coc, A. 2006, in International Symposium on Nuclear Astrophysics - Nuclei in the Cosmos. X.

(CERN, European Organization for Nuclear Research), p. 11

Davé, R., Cen, R., Ostriker, J. P., Bryan, G. L., Hernquist, L., Katz, N., Weinberg, D. H., Norman, M. L., \& O'Shea, B. 2001, ApJ, 552, 473

Green, J. C. 2001, Proc. SPIE, 4498, 229

Hernandez-Monteagudo, C., Trac, H., Verde, L., \& Jimenez, R. 2006, ApJ, 652, L1

Hiyashi, E., \& Navarro, J. N. 2007, MNRAS, 373, 1117

Impey, C. D., Petry, C. E., \& Flint, K. P. 1999, ApJ, 524, 536

Kamionkowski, M., Jungman, G., Kosowsky, A. \& Spergel, D. N. 1995, in S. Holt \& F. Sonneborn (eds.), Sixth Annual Octboer Astrophysics Conference, ASP Conference Series (San Francisco: ASP), vol. 99, p. 74

Kawahara, H., Yoshikawa, K, Sasaki, S., Suto, Y., Kawai, N., Mitsuda, K., Ohashi, T., \& Yamasaki, N. 2006, PASJ, 58, 657

Lehner, N., Savage, B. D., Richter, P., Sembach, K. R., Tripp, T. M., \& Wakker, B. P. 2007, ApJ, 658, 680

Martin, C. L. 2006, in J. W. Mason (ed.) Astrophysics Update 2, (Heidelberg: Springer Verlag), p. 337

McGaugh, S. S., Rubin, V. C., \& de Blok, W. J. G. 2002, AJ, 122, 2381

McLin, K. M., Stocke, J. T., Weymann, R. J., Penton, S. V., \& Shull, M. J. 2002, ApJ, 574, 115

Moore, B., Ghigna, S., Governato, F., Lake, G., Quinn, T., Stadel, J., \& Tozzi, P. 1999, ApJ, 524,19

Penton, S. V., Shull, M. J., \& Stocke, J. T. 2000, ApJ, 544, 150

Penton, S. V., Stocke, J. T., \& Shull, M. J. 2002, ApJ, 565, 720

Petitjean, P., \& Charlot, S. (eds.) Structure and Evolution of the Intergalactic Medium from QSO Absorption Line Systems (Paris: Editions Frontières)

Prochaska, J. X., Wolfe, A. M., Howk, J. C., Gawsier, E., Burles, S. M. \& Cooke, J. 2007, ApJSuppl, 171, 29

Rassera, Y., \& Teyssier, R. 2006, A\&A, 445, 1

Rauch, M., Miralda-Escude, J., Sargent, W., Barlow, T., Weinberg, D., Hernquist, L., Katz, N., Cen, R., \& Ostriker, J. 1997, ApJ, 489, 7

Scott, J., Bechtold, J. B., Morita, M., Dobrzycki, A., \& Kulkarni, V. 2002, ApJ, 571, 665

Sembach, K. R., Wakker, B. P., Savage, B. D., Richter, P., Meade, M., Shull, J. M., Jenkins, E. B., Sonneborn, G., \& Moos, H. W. 2002, ApJS, 146, 165

Shull, M. J., Penton, S. V., Stocke, J. T., Giroux, M. L., van Gorkom, J. H., Lee, Y. H., \& Carilli, C. 1998, AJ, 116, 2094

Stocke, J. T., Penton, S. V., Danforth, C. W., Shull, M. J., Tumlinson, J., \& McLin, K. M. 2006, ApJ, 641, 217

Tripp, T. M. et al. 2002, ApJ, 575, 679

Tripp, T. M, Sembach, K. R., Bowen, D. V., Savage, B. D., Jenkins, E. B., Lehner, N., \& Richter, P. 2007, ApJSuppl, in press, astro-ph07061214

Wang, Q. D., Yao, Y., Tripp, T. M., Fang, T.-T., Cui, W., Nicastro, F., Mathur, S., Williams, R. J., Song, L., \& Croft, R. 2005, ApJ, 635, 386

Wakker, B. P., Savage, B. D., Sembach, K. R., Richter, P., \& Fox, A. J. 2005, in R. Braun (ed.) Extra-Planar Gas ASP Conference Series (San Francisco: ASP), vol. 331, p. 11

Zhang, Y., Anninos, P., Norman, M. L., \& Meiksin, A. 1997, ApJ, 485, 496

Zwaan, M., Meyer, M. J, Staveley-Smith, L., and Webster, R. L. 2005 MNRAS, 359, 30 